\title{
INFLUÊNCIA DA ESTRUTURA DE AUDITORIA, CONSELHO DE ADMINISTRAÇÃO E QUALIDADE DA INFORMAÇÃO CONTÁBIL NO ÍNDICE MARKET TO BOOK VALUE DE EMPRESAS BRASILEIRAS LISTADAS NA B3
}

\author{
THE INFLUENCE OF AUDIT CAPABILITY, CORPORATE BOARD AND QUALITY OF \\ ACCOUNTING INFORMATION ON THE INDEX MARKET TO BOOK VALUE OF BRAZILIAN \\ COMPANIES LISTED ON B3
}

\author{
Andréia Carpes Dani ${ }^{1}$ \\ Universidade Regional de Blumenau \\ andreiacarpedani@gmail.com \\ Edgar Pamplona \\ Universidade Regional de Blumenau \\ edgarpamplona@hotmail.com \\ Paulo Roberto da Cunha \\ Universidade Regional de Blumenau \\ pauloccsa@furb.br
}

\begin{abstract}
RESUMO
Objetivo: Verificar a influência da estrutura de auditoria, conselho de administração e qualidade da informação contábil no índice Market to Book Value de empresas brasileiras listadas na B3.

Fundamento: A estrutura de auditoria e o conselho de administração podem influenciar o desempenho de mercado das empresas (Moutinho, Cerqueira e Brandão, 2012). Além disso, a qualidade da informação contábil tende ser maximizada por mecanismos de auditoria bem definidos, mitigando práticas de manipulação e proporcionando maior transparência aos usuários da informação contábil, o que deve ser identificado pelo mercado e refletir no desempenho da empresa (Almeida \& Almeida, 2009; Politelo, 2013).

Método: Pesquisa descritiva, quantitativa e documental. Os dados foram extraídos da base Economática ${ }^{\circledR}$ e de formulários de referência. O período de estudo é de 2012 a 2014, tendo a amostra 135 empresas. A análise dos dados foi realizada pela regressão linear múltipla.

Resultados: Têm-se que reputação da auditoria independente e honorários pagos de não auditoria possuem relação positiva com o desempenho de mercado, enquanto presença do comitê de auditoria e dualidade de diretor estão negativamente relacionados com a performance corporativa. Finalmente, tem-se ainda que empresas que gerenciam mais resultados (como proxy da qualidade da informação contábil) apresentam Market to Book Value superior.
\end{abstract}

Artigo recebido em: 13/09/2018. Revisado por pares em: 11/12/2018. Reformulado em: 11/01/2019. Recomendado para publicação em: 28/03/2019 por Vagner Antônio Marques (Editor Adjunto). Publicado em: 26/05/2019. Organização responsável pelo periódico: UFPB

${ }^{1}$ Endereço: Rua Antônio da Veiga, 140 - Sala C 202 - Bairro Victor Konder. CEP: 89012-900 - Blumenau/SC - Brasil. 
Contribuições: Amplia a perspectiva de gestores corporativos, no âmbito da estrutura de auditoria, conselho de administração e qualidade da informação contábil, a fim de maximizar o valor das empresas que administram, sendo isto, notadamente, um de seus mais importantes enfoques administrativos.

Palavras-chave: Market to Book Value. Estrutura de Auditoria. Conselho de Administração. Qualidade da Informação Contábil. Empresas Brasileiras.

\section{ABSTRACT}

Objective: To verify the influence of the audit structure, corporate board and the quality of accounting information in the Market to Book Value index of Brazilian companies listed in B3.

Background: The audit structure and corporate board can influence the market performance of companies (Moutinho, Cerqueira and Brandão, 2012). In addition, the quality of accounting information tends to be maximized by well-defined audit mechanisms, mitigating manipulation practices and providing greater transparency to users of accounting information, which should be identified by the market and reflect on the company's performance (Almeida \& Almeida, 2009; Politelo, 2013).

Method: Descriptive, quantitative and documentary research. Data were extracted from Economática ${ }^{\circledR}$ database and reference forms. The study period is from 2012 to 2014 , with the sample 135 companies. Data analysis was performed by multiple linear regression.

Results: It has been that independent audit reputation and paid non-audit fees possess positive relation with market performance, while audit committee attendance and duality are negatively related to corporate performance. Finally, companies that manage more results (as a proxy for the quality of accounting information) have a higher Market to Book Value.

Contributions: It broadens the perspective of corporate managers, within the scope of the audit structure, corporate board and quality of accounting information, in order to maximize the value of the companies they manage, being, in particular, one of its most important administrative approaches.

Keywords: Market to Book Value. Audit structure. Corporate board. Quality of accounting information. Brazilian companies.

\section{INTRODUÇÃO}

As estruturas de auditoria e do conselho de administração podem estar relacionadas com diversos indicadores, entre eles os de desempenho de mercado. Neste sentido, Ramos e Martinez (2006) relatam que a adoção de mecanismos de governança corporativa - como é o caso da auditoria e do conselho de administração -, são considerados como uma condição essencial para o desenvolvimento do mercado de capitais. A boa governança corporativa deve estimular adequadamente os gestores a perseguir objetivos que sejam do interesse da companhia e de seus acionistas, visando otimizar o retorno sobre investimentos e o crescimento a longo prazo (Ramos \& Martinez, 2006). Essencialmente, os mecanismos de auditoria proporcionam maior transparência e melhoria da qualidade da informação contábil, auxiliando na mitigação de práticas de manipulação contábil, além de contribuir positivamente para o desempenho empresarial (Almeida \& Almeida, 2009; Politelo, 2013).

No conselho de administração observando-se aspectos quantitativos e qualitativos ao que se refere à qualificação, independência, avaliação e remuneração, qualidade da informação, entre inúmeros outros (Ramos \& Martinez, 2006). De fato, conforme Silveira, Barros e Famá (2003), o conselho de administração é considerado um dos principais mecanismos da governança corporativa. Desta forma, pesquisas como de Ujunwa (2012), Gaur, Bathula e Singh (2015) e Wahba (2015) tem buscado verificar a influência da governança corporativa no desempenho das companhias considerando variáveis relacionadas às características do conselho de administração. 
Ademais, no que concerne aos mecanismos de auditoria, diversas variáveis acerca da estrutura de auditoria têm sido relacionadas com o desempenho das organizações. Neste sentido, Al Farooque, Zijl, Dunstan e Karim (2007), Haat, Rahman e Mahenthiran (2008), Christensen, Kent e Stewart (2010), Azim (2012), Moutinho, Cerqueira e Brandão (2012), Politelo (2013) e Martinez e Moraes (2014) utilizaram variáveis como reputação da empresa de auditoria independente, presença de comitê de auditoria independente nas organizações, honorários pagos aos auditores independentes a título de serviços de auditoria, bem como serviços adicionais (de não auditoria), entre outras.

É comum ainda abordagens que determinam a estrutura de auditoria e do conselho de administração como difusores de informações contábeis de melhor qualidade (Ramos e Martinez, 2006). Nesse contexto, é importante mencionar a relevância da qualidade da informação contábil, pois esta influencia na alocação de recursos bem como na distribuição de riqueza entre os diversos agentes econômicos nas organizações (Paulo \& Leme, 2009). Porém, os administradores também podem utilizar do julgamento sob a informação financeira para alterar ou iludir os usuários externos acerca do desempenho da companhia através do gerenciamento de resultados (Healy \& Wahlen, 1999), sugerindo possível comportamento dúbio desta variável para a performance corporativa.

Por fim, quanto ao índice market to book, sendo uma medida de desempenho de mercado corporativo, este é utilizado como a relação entre o valor econômico da empresa capturado pelo mercado sobre o valor contábil. A partir de incentivos, pode-se considerar que "uma empresa para manter seu valor de mercado maior que seu valor contábil precisa apresentar resultados para criar expectativas de fluxo de caixa futuro aos seus acionistas e agentes com algum interesse na firma" (Gilio, 2010). Mais precisamente, tem-se que empresas com este índice elevado indicam que os agentes possuem alta expectativa por seus resultados futuros (fluxo de caixa e retorno das ações), ou seja, o mercado reconhece informações que a contabilidade não pode ou não consegue evidenciar devido aos princípios e/ou pelos dispositivos legais (Almeida, Lopes \& Corrar, 2011).

Frente ao exposto, esta pesquisa busca responder a seguinte questão de pesquisa: qual a influência da estrutura de auditoria, conselho de administração e qualidade da informação contábil no índice market to book value de empresas brasileiras listadas na Brasil, Bolsa, Balcão (B3)? Consequentemente, o estudo tem como objetivo verificar a influência da estrutura de auditoria, conselho de administração e qualidade da informação contábil no índice market to book value de empresas brasileiras listadas na B3.

Esta pesquisa se justifica por inúmeros fatores. Neste sentido, no que concerne a evidências empíricas anteriores, Politelo (2013) verificou relação positiva entre os mecanismos de governança corporativa e o desempenho de empresas familiares listadas na BM\&FBovespa, considerando também variáveis relacionadas a auditoria. No que tange a relação entre qualidade da informação contábil e o índice market to book, não foram encontrados estudos anteriores no cenário brasileiro considerando o escopo totalitário proposto, tendo esta pesquisa, portanto, anseios teóricos e metodológicos mais robustos.

Complementarmente, Martinez (2010) explica que o papel do conselho de administração e a formulação dos serviços de auditoria independente fortalecem as práticas de governança. Ademais, tem-se também que o perfil do conselho de administração e das empresas de auditoria independente pode influenciar na qualidade da informação contábil. De modo geral, estudos supracitados verificaram isoladamente que mecanismos de governança estão ligados à qualidade da informação contábil. Adicionalmente, esta pesquisa contribui no sentido de verificar a influência da qualidade da informação sobre o desempenho de mercado das empresas brasileiras.

Finalmente, o estudo se justifica ainda pela relevância do tema para a literatura da área, bem como para os stakeholders, visto que o índice market to book constitui-se como um incentivo do mercado, por meio do qual os executivos procuram atender as expectativas dos acionistas. Além disso, destaca-se que o valor econômico da empresa no mercado é considerado como uma medida não 
amplamente entendida (Gilio, 2010) e que, portanto, merece melhor investigação no que concerne sua relevância para a avaliação do desempenho das companhias.

\section{FUNDAMENTAÇÃO TEÓRICA}

\subsection{Estrutura de Auditoria e o Índice Market to Book Value}

As medidas de desempenho de mercado possuem abrangente importância no mercado de capitais, estando entre elas, com destaque, o índice Market to Book Value. Conforme Firth (1997), tais medidas relacionam-se com as estimativas de lucro das organizações e suas taxas de crescimento, o que permite Politelo (2013) relatar que estas refletem a concepção que o mercado possui da empresa. Mais especificamente acerca do índice Market to Book Value, Chen e Zhao (2006) entendem que este mede as oportunidades de crescimento das organizações, refletindo assim as expectativas do mercado acerca dos fluxos de caixa futuros que a firma pode gerar.

Quanto ao desempenho da empresa, medido por indicadores contábeis e/ou de mercado, Moutinho et al. (2012) relatam que a estrutura de auditoria pode influenciar tais indicadores, como no caso o índice de mercado Market to Book Value. Mais precisamente, diversas são as variáveis estudadas que podem afetar o desempenho das organizações, como a reputação da empresa de auditoria independente, a presença de comitê de auditoria nas organizações e os honorários pagos aos auditores independentes por serviços de auditoria e não auditoria.

Neste contexto, tem-se inicialmente a reputação da empresa de auditoria independente, sendo um dos aspectos mais abordados na literatura, que procura comparar o resultado de organizações auditadas por empresas de auditoria big four (Deloitte, Ernst Young, PricewaterhouserCoopers e KPMG) com as não big four. Conforme Lameira (2007) e Collin, Smith, Umans, Broberg e Tagesson (2013), o fato de uma organização contar com a revisão de uma das maiores empresas de auditoria (no caso, as big four) tende a aumentar o monitoramento, bem como a expertise dos prestadores de serviço e, por consequência, o desempenho da organização tomadora é maximizado

No campo empírico, Al Farooque et al. (2007) testaram, com amostra de empresas de Bangladesh, entre outros fatores, se existe influência da reputação da auditoria independente no desempenho das organizações do país. Seus achados confirmaram tal relação, havendo, portanto, influência positiva e significativa entre a reputação da firma de auditoria e o desempenho das organizações bangladeshianas, corroborando, portanto, com os preceitos teóricos existentes. Por fim, os autores (2007) ainda salientam que a qualidade da contabilidade e da auditoria das empresas são mecanismos de controles vitais para proteger os interesses dos acionistas externos. Com base nestes preceitos, emerge-se a seguinte hipótese de pesquisa:

$H_{1}$ - Existe influência positiva entre a reputação da empresa de auditoria independente e o índice de desempenho de mercado Market to Book Value de empresas brasileiras listadas na B3.

Posteriormente, tem-se a abordagem acerca da presença do comitê de auditoria nas organizações. Neste sentido, Davidson, Stewart e Kent (2005) relatam que o conselho de administração geralmente delega responsabilidades de relatórios financeiros ao comitê de auditoria e, por consequência, o referido comitê passa a ser o mecanismo mais adequado para proporcionar aos acionistas maior proteção e manter a qualidade das demonstrações financeiras. Neste cenário, Christensen et al. (2010) e Azim (2012) determinam que os comitês de auditoria desempenham papel fundamental, pois tem potencial para diminuir a frequência de problemas nos relatórios financeiros das empresas. Com isso, mediante ao conhecimento de que comitês de auditoria proporcionam transparência, a tendência é que estes possam contribuir positivamente para o desempenho das organizações (Politelo, 2013), incluindo o de mercado. Visto isto, a hipótese a ser testada perpassa por: 
$H_{2}$ - Existe influência positiva entre a presença do comitê de auditoria nas organizações e o índice de desempenho de mercado Market to Book Value de empresas brasileiras listadas na B3.

Por fim, acerca dos honorários pagos aos auditores independentes, Francis (1984) relata que preços mais altos despendidos com honorários são consistentes com auditorias de qualidade superior (Francis, 1984). Desta forma, tais honorários podem ser usados para analisar a qualidade das auditorias e se existe demanda por diferenciação no mercado (Hallak \& Silva, 2012). De fato, a teoria da agência aborda que algumas empresas possuem incentivos econômicos para exigirem auditorias de qualidade superior a fim de monitorar contratos, visando, sobretudo, minimizar os custos de agência (Francis, 1984).

Neste sentido, diversos estudos têm buscado relacionar os honorários de auditoria, que podem ser entendidos como um fator que afeta a qualidade das auditorias, com o desempenho das organizações. Assim, corroborando com os argumentos teóricos apresentados até o momento, Martinez e Moraes (2014), no contexto brasileiro, relacionaram os honorários de auditoria contábil com o desempenho de mercado das empresas, medido pela proxy $Q$ de Tobin e encontraram associação positiva entre ambos.

Contudo, no outro extremo, há autores que trazem entendimento oposto desta relação, como Haat et al. (2008) e Moutinho et al. (2012), relatando que empresas com baixo desempenho pagam honorários de auditoria relativamente mais elevados em comparação a empresas com bom desempenho. Isto se deve ao fato que empresas com desempenho insatisfatório investem mais em honorários de auditoria a fim de melhorar seus resultados, buscando superar a fraca gestão que vem sistematicamente prejudicando a performance de organizações que se encontram em tal situação. Empiricamente, os próprios estudos de Haat et al. (2008) e Moutinho et al. (2012), sendo o primeiro com empresas da Malásia e o segundo com empresas norte-americanas forneceram evidências empíricas que há relação negativa entre honorários de auditoria e desempenho.

Além disso, há outro fator importante a ser estudado, que corresponde aos serviços de não auditoria prestados por empresas de auditoria independente, que abrangem principalmente trabalhos de consultoria. Neste sentido, Whisenant, Sankaraguruswamy e Raghunandan (2003) destacam que pesquisas têm mostrado grande interesse nos serviços de não auditoria, tendo-se evidências de que serviços de auditoria e de não auditoria são determinados conjuntamente. No ínterim, Dickins, Higgs e Skantz (2008) revelam que existe por parte de inúmeros clientes o interesse em tomar serviços de não auditoria de seus auditores independentes, todavia, apesar da existência de pesquisas que consideram honorários tanto de auditoria como de não auditoria cobrados, ainda não há consenso da relação destes serviços, sobretudo quanto ao sinal, com outras variáveis contábeis, como as de desempenho. Diante da abordagem teórica e empírica apresentada acerca dos honorários pagos aos auditores independentes tanto por serviços de auditoria como de não auditoria, elaboram-se as seguintes hipóteses de pesquisa, conforme segue:

$H_{3}$ - Existe influência entre os honorários pagos aos auditores independentes e o índice de desempenho de mercado Market to Book Value de empresas brasileiras listadas na B3.

$H_{3 a}$ - Existe influência entre os honorários pagos aos auditores independentes referente aos serviços de auditoria e o índice de desempenho de mercado Market to Book Value de empresas brasileiras listadas na B3.

$H_{3 b}$ - Existe influência entre os honorários pagos aos auditores independentes referente aos serviços de não auditoria e o índice de desempenho de mercado Market to Book Value de empresas brasileiras listadas na B3. 


\subsection{Conselho de Administração e o Índice Market to Book Value}

A qualidade da governança corporativa fortalece os controles da entidade levando a minimização da assimetria informacional e dos custos de agência (Oliveira, Paulo \& Martins, 2013). Assim, o conselho de administração, como parte essencial do sistema de governança corporativa, converge para o aumento do valor preditivo da informação contábil, cuja consequência é permitir uma empresa mais confiável por parte de novos investidores, elevando o valor de mercado corporativo. A implantação do conselho de administração como boas práticas de governança contribui “para melhorar a dinâmica do funcionamento empresarial, o que provavelmente possibilitará aumento de eficiência, queda de riscos e, por consequência, melhoria na avaliação das empresas pelo mercado e investidores" (Sousa, 2014).

Silveira, Barros e Famá (2006) ratificam que quase à totalidade das discussões sobre governança corporativa partem do pressuposto que "os mecanismos de governança influenciam o desempenho das empresas" (Silveira et al., 2006), maximizando-o, o que é convergente também quando analisados especificamente as características do conselho de administração. Portanto, a governança corporativa busca o fortalecimento do mercado acionário, visando minimizar possíveis conflitos de interesses entre acionistas minoritários e majoritários e, gerando, consequentemente, maior retorno às partes interessadas na organização (Nascimento \& Reginato, 2008).

Em suma, perspectivas teóricas anteriores demonstram que boas práticas de governança aumentam o desempenho das empresas (Almeida, Santos, Ferreira e Torres, 2010). Para aferição destas boas práticas de governança, diversos fatores podem ser considerados (Silveira \& Barros, 2008; Correia, Amaral e Louvet, 2011). Mediante literatura, dentre tais tem-se o tamanho do conselho de administração, a proporção de mulheres no conselho de administração, a dualidade de função do diretor (este, em exceção aos demais, sendo um fator negativo às boas práticas de governança), o número de reuniões do conselho de administração e a expertise do conselho de administração.

Observando cada um destes individualmente, tem-se inicialmente a discussão acerca do tamanho do conselho de administração. Johl, Kaaur e Cooper (2015) mencionam que com o intuito de determinar o impacto em seus resultados, o tamanho do conselho de administração das corporações deve abranger número suficiente de membros para que se possa operar em equipe com eficiência (Johl et al., 2015). Apesar da não convergência por completo, o levantamento de Johl et al. (2015) aponta que majoritariamente as evidências prévias apresentam relação positiva entre o tamanho do conselho de administração e o desempenho corporativo, sendo inclusive convergente com as evidências recentes destes autores no cenário da Malásia. Logo, tem-se a seguinte hipótese de pesquisa:

$H_{4}$ - Existe influência positiva entre o tamanho do conselho de administração e o índice de desempenho de mercado Market to Book Value de empresas brasileiras listadas na B3.

Ademais, Campbell e Minguez-Vera (2008), partindo do entendimento de que o papel de monitoramento do conselho de administração é um importante mecanismo de controle no cerne da governança corporativa, focaram seu estudo na questão de composição de gênero do conselho, visto que esta configuração pode afetar a qualidade do monitoramento e, consequentemente, o desempenho da empresa. O diferencial de pesquisa perseverou pelo contexto estudado, no caso a Espanha, que historicamente teve participação feminina mínima na força de trabalho, mas que vem introduzindo legislação para melhorar a igualdade de oportunidades entre gêneros. Os resultados, em suma, sugerem que a maior diversidade de gêneros (ou seja, o equilíbrio das mulheres no cargo) no conselho gera ganhos econômicos. Assim, especificamente, tem-se que a maior porcentagem de mulheres no quadro tem um efeito positivo no valor da empresa. Com base nestes fundamentos, a hipótese a ser testada condiz com: 
$H_{5}$ - Existe influência positiva entre a proporção de mulheres no conselho de administração e o índice de desempenho de mercado Market to Book Value de empresas brasileiras listadas na B3.

Ehikioya (2009), pautado em estudos anteriores, menciona que a estrutura de governança corporativa, medida por fatores como estrutura de propriedade, composição do conselho, tamanho do conselho, dualidade do CEO (Chief Executive Officer), entre outros, tem forte influência no desempenho das empresas. Neste sentido, no cenário nigeriano, desenvolveu pesquisa empírica com foco na variável dualidade do CEO. Os achados da pesquisa asseveram que há evidências significativas para apoiar que a dualidade do CEO, de fato, afeta negativamente a performance corporativa. Como contribuição principal, tem-se o fornecimento de informações úteis para diversas partes interessadas no assunto, como, por exemplo, formuladores de políticas e acadêmicos. Logo, para a amostra deste estudo, espera-se, conforme hipótese, a seguinte relação:

$H_{6}$ - Existe influência negativa entre a dualidade de função do diretor e o índice de desempenho de mercado Market to Book Value de empresas brasileiras listadas na B3.

Argumenta-se que as reuniões do conselho de administração e a participação efetiva dos membros em tais reuniões são canais importantes dos quais os conselheiros obtêm informações específicas e essenciais capazes de fazer com que estes cumpram sua função de monitoramento nas corporações (Johl et al., 2015), função esta que se corretamente desenvolvida, tende a maximizar a performance corporativa. Empiricamente, em estudo conduzido com 169 empresas sul-africanas de capital aberto, Ntim e Osei (2011) verificaram relação positiva entre a frequência de reuniões do conselho de administração e o desempenho corporativo naquele país. Vinculados a estes aspectos teóricos e empíricos, tem-se, na sequência, a elaboração da seguinte hipótese de pesquisa:

$H_{7}$ - Existe influência positiva entre o número de reuniões do conselho de administração e o índice de desempenho de mercado Market to Book Value de empresas brasileiras listadas na B3.

Por fim, de acordo com Johl et al. (2015), é de suma importância que especialistas estejam presentes para assegurar maior responsabilidade acerca da ampla gama de questões pertencentes ao conselho de administração (Johl et al., 2015). Notadamente, tal especialização geralmente perpassa por profissionais formados em ciências contábeis, administração e economia, por possuírem conhecimentos acerca dos demonstrativos financeiros e nas técnicas de finanças. Empiricamente, diversos estudos em todo o mundo têm atestado existência de relação positiva entre a expertise dos membros do conselho de administração e o desempenho corporativo, como, por exemplo, Johl et al. (2015) que analisaram 731 empresas malaias com informações ano de 2009. Perante isto, estendendo o conhecimento a ser testado no Brasil, tem-se a seguinte hipótese de pesquisa:

$H_{8}$ - Existe influência positiva entre a expertise do conselho de administração e o índice de desempenho de mercado Market to Book Value de empresas brasileiras listadas na B3.

\subsection{Qualidade da Informação Contábil e o Índice Market to Book Value}

A preocupação com a qualidade da informação contábil vem sendo foco de debate há bastante tempo, visto que estudos utilizando modelos de gerenciamento de resultados (sendo uma das métricas para a qualidade da informação contábil) iniciaram na década de 1980. Neste sentido, destaca-se que a qualidade da informação contábil tem sido, em muitos estudos, estritamente vinculada com a qualidade da auditoria. DeAngelo (1981) relata que se espera de uma auditoria de qualidade 
detectar falhas no sistema contábil de seu cliente (se houver) e, por sequência, reportar essas falhas ao mercado.

No âmago da discussão, Watts e Zimmerman (1981) trazem à tona questões sobre a teoria da agência, alertando que um conjunto específico de custos surge quando há conflito de interesses entre as partes que tenham ligação com a empresa. Desta forma, relatam que demonstrações financeiras e auditoria possuem potencial de atenuar tais conflitos de interesse, diminuindo a assimetria informacional entre as partes interessadas. Contudo, Watts e Zimmerman (1981) alertam que é improvável supor que os auditores são perfeitamente independentes de seus clientes. $\mathrm{O}$ auditor, no anseio da empresa, pode perder a independência quando passa a ter interesses e, assim, a probabilidade condicional que irá relatar uma violação torna-se, por consequência, menor.

Diante deste contexto, Lopes e Iudícibus (2012) relatam que, para o auditor, quando é razoável supor que as perdas associadas à aceitação de um cliente sejam inferiores as perdas derivadas de ter sua imagem vinculada a um "escândalo" contábil, o auditor tem motivos para reportar as falhas detectadas e, portanto, ser independente. Contudo, quando o cenário se inverte, é razoável supor que o auditor possa vir a perder independência, não divulgando as falhas detectadas no processo de auditoria.

Apesar das dificuldades existentes de se dimensionar a qualidade da auditoria, estudos vêm, ao longo do tempo, buscando vincular fatores observáveis que de alguma forma possam representar o referido fenômeno. Notadamente, a partir da década de 1990, métricas baseadas em informações contábeis passaram a ser amplamente utilizadas para medir a qualidade das auditorias, advindas, sobretudo, de modelos de gerenciamentos de resultados, como o de Kang e Sivaramakrishnan (1995), que utiliza os accruals discricionários como proxy de gerenciamento de resultados.

Para Lopes e Iudícibus (2012), questões acerca da qualidade das auditorias em consonância com a própria qualidade da informação contábil, apontam que o caminho até então percorrido pela literatura teórica e empírica é bastante intuitivo: quanto maior (ou melhor) a qualidade de uma auditoria, maior será a qualidade de uma informação contábil. Partindo deste entendimento e, com vistas ao conhecimento anterior de que há relação entre a estrutura de auditoria e o desempenho das organizações, espera-se que a qualidade da informação contábil, medida pelos accruals discricionários e vinculada à estrutura de auditoria, tenha relação com o desempenho de mercado das organizações. Logo, emerge-se a hipótese a seguir apresentada:

$H_{9}$ - Existe influência entre a qualidade da informação contábil e o índice de desempenho de mercado Market to Book Value de empresas brasileiras listadas na B3.

\section{PROCEDIMENTOS METODOLÓGICOS}

Com o intuito de verificar a influência da estrutura de auditoria, conselho de administração e qualidade da informação contábil no índice Market to Book Value de empresas brasileiras listadas na B3, a presente pesquisa é delineada como descritiva, com abordagem quantitativa e análise documental. A população do estudo corresponde a todas as empresas brasileiras de capital aberto listadas na B3. Posteriormente, retiraram-se da pesquisa as organizações financeiras por apresentarem características particulares, as empresas que não dispunha de todas as informações para cálculo das variáveis necessárias no estudo e, ainda, aquelas que tiveram informações consideradas outliers, por estarem mais de dois desvios-padrões distantes da média (nas variáveis numéricas). Desta forma, amostra ficou composta por 135 empresas e o período de análise é de 2012 a 2014, gerando assim ao todo 405 observações.

O modelo aplicado para identificação dos acrruals é o KS, desenvolvido por Kang e Sivaramakrishnan (1995). Conforme Healy e Wahlen (1999), o KS configura-se no mais robusto modelo de 
gerenciamento de resultados. De forma mais abrangente, Martinez $(2001 ; 2008)$ relata que embora muito se utilize o modelo de Jones para estimar os accruals, a melhor alternativa é o modelo KS, pois o mesmo possui potencial para mitigar problemas como de erros nas variáveis, variáveis omitidas e simultaneidade que existe no modelo de Jones. Assim, Martinez $(2001 ; 2008)$ enfatiza que o modelo KS representa expressivo avanço sobre o modelo de Jones. O modelo desenvolvido por Kang e Sivaramakrishnam (1995) é, portanto, constituído conforme a seguir:

$$
\frac{A B_{t}}{A T_{t-1}}=\beta_{0}+\beta_{1}\left[\frac{\delta_{1} R E V_{t}}{A T_{t-1}}\right]+\beta_{2}\left[\frac{\delta_{2}-E X P_{t}}{A T_{t-1}}\right]+\beta_{3}\left[\frac{\delta_{3}+G P P E_{t}}{A T_{t-1}}\right]+\varepsilon
$$

Equação (1)

$A B_{t}=$ Accrual balance (acumulações totais) no período $t$;

$=A R+I N V+O C A-C L-D E P$.

$A R_{t}=$ Contas a receber, excluindo impostos a compensar no período $t$;

$I N V_{t}=$ Estoques no período $t$;

$O C A_{t}=$ Outros ativos circulantes excluindo caixa, contas a receber e estoques no período $t$;

$C L_{t}=$ Passivos circulantes excluindo impostos a pagar e parcelas de dívidas de longo prazo no circulante no período $t$;

$D E P_{t}=$ Depreciação no período $t$;

$R E V_{t}=$ Receita líquida no período $t$;

$E X P_{t}=$ Despesas operacionais antes da depreciação no período $t ;$

$G P P E_{t}=$ Permanente (imobilizado) no período $t ;$

$A T_{t}=$ Ativos totais no início do período, ou seja, $t-1$;

$\delta_{1}=A R_{t-1} / R E V_{t-1}$

$\delta_{2}=I N V_{t-1}+O C A_{t-1}-C L_{t-1} / E X P_{t-1} ;$

$\delta_{3}=D E P_{t-1} / G P P E_{t-1}$

$\varepsilon=$ Resíduos da regressão, acumulação discricionária.

Diante da equação 1 apresentada, tem-se então os accruals discricionários, que são utilizados em termos absolutos como a última das variáveis independentes da equação 2 (abaixo). Neste sentido, destaca-se que o sinal dos accruals torna-se irrelevante na análise, pois independentemente se os resultados estão sendo gerenciados de forma negativa ou positiva, existe perda na qualidade de tais informações. Krishnan (2003) relata que se deve considerar os accruals em termos absolutos, porque quanto maior seu grau, mais elevado é a flexibilidade sobre a contabilidade que as auditorias independentes permitem a seus clientes, o que reduz, por consequência, a qualidade da informação.

Ademais, especificamente acerca da equação 2, esta possui como variável dependente o desempenho de mercado, que compreende ao índice Market to Book Value e, como variáveis independentes, têm-se aquelas vinculadas a estrutura de auditoria, conselho de administração e qualidade da informação contábil. Desta forma, antes de expor a equação 2, faz-se necessário apresentar o Quadro 1 das variáveis utilizadas. 
Quadro 1 - Constructo das Variáveis

\begin{tabular}{|c|c|c|c|c|}
\hline \multicolumn{2}{|c|}{ Dimensão } & Variáveis & Operacionalização & Fonte \\
\hline \multicolumn{2}{|c|}{$\begin{array}{l}\text { Var. Dependente - } \\
\text { Desempenho de Mer- } \\
\text { cado }\end{array}$} & Market to Book Value (MTB) & $\frac{\text { Valor de Mercado da Empresa }}{\text { Patrimônio Líquido Contábil }}$ & Economática ${ }^{\circledR}$ \\
\hline \multirow{10}{*}{ 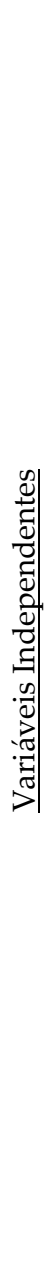 } & \multirow{4}{*}{$\begin{array}{l}\text { Estrutura de Au- } \\
\text { ditoria }\end{array}$} & $\begin{array}{l}\text { Reputação da Auditoria In- } \\
\text { dependente (RepAud) }\end{array}$ & $\begin{array}{l}\text { Variável dummy, } 1 \text { se a empresa é au- } \\
\text { ditada por Big Four, } 0 \text { caso contrário. }\end{array}$ & $\begin{array}{l}\text { Formulário de re- } \\
\text { ferência - item } 2.1\end{array}$ \\
\hline & & $\begin{array}{l}\text { Presença de Comitê de Audi- } \\
\text { toria (ComAud) }\end{array}$ & $\begin{array}{l}\text { Variável dummy, } 1 \text { se existe Comitê } \\
\text { de Auditoria, } 0 \text { caso contrário. }\end{array}$ & $\begin{array}{l}\text { Formulário de re- } \\
\text { ferência - item } \\
12.7 \\
\end{array}$ \\
\hline & & $\begin{array}{l}\text { Honorários Pagos de Audito- } \\
\text { ria (HAud) }\end{array}$ & $\frac{\text { Honorários Pagos de Auditoria }}{\text { Ativo Total }_{\mathrm{t}}}$ & $\begin{array}{l}\text { Formulário de re- } \\
\text { ferência - item } 2.1 \\
\text { e Economática }{ }^{\circledR}\end{array}$ \\
\hline & & $\begin{array}{l}\text { Honorário Pagos de Não Au- } \\
\text { ditoria (HNAud) }\end{array}$ & $\frac{\text { Honorários Pagos Não Auditoria }}{\text { Ativo Total }_{\mathrm{t}}}$ & $\begin{array}{l}\text { Formulário de re- } \\
\text { ferência - item } 2.1 \\
\text { e Economática }{ }^{\circledR}\end{array}$ \\
\hline & \multirow{5}{*}{$\begin{array}{l}\text { Conselho de Ad- } \\
\text { ministração }\end{array}$} & $\begin{array}{l}\text { Tamanho do Conselho de } \\
\text { Administração (TamCA) }\end{array}$ & $\begin{array}{l}\text { Log do número total de membros no } \\
\text { conselho. }\end{array}$ & $\begin{array}{l}\text { Formulário de re- } \\
\text { ferência - } \\
\text { item } 13.2 \\
\end{array}$ \\
\hline & & $\begin{array}{l}\text { Gênero do Conselho de Ad- } \\
\text { ministração (GenCA) }\end{array}$ & $\begin{array}{l}\text { A relação entre o número de mulhe- } \\
\text { res no conselho e o número total de } \\
\text { conselheiros. }\end{array}$ & $\begin{array}{l}\text { Formulário de re- } \\
\text { ferência - } \\
\text { item } 12.6 / 8 \\
\end{array}$ \\
\hline & & $\begin{array}{l}\text { Dualidade do Diretor (Du- } \\
\text { alD) }\end{array}$ & $\begin{array}{l}\text { Variável dummy, } 1 \text { se o CEO é o pre- } \\
\text { sidente do conselho de administra- } \\
\text { ção, } 0 \text { caso contrário. }\end{array}$ & $\begin{array}{l}\text { Formulário de re- } \\
\text { ferência - } \\
\text { item } 12.6 / 8 \\
\end{array}$ \\
\hline & & $\begin{array}{l}\text { Reuniões do Conselho de Ad- } \\
\text { ministração (ReunCA) }\end{array}$ & $\begin{array}{l}\text { Número total de reuniões do conse- } \\
\text { lho no ano. }\end{array}$ & $\begin{array}{l}\text { Inf. Relevantes - } \\
\text { Reunião da Ad- } \\
\text { ministração }\end{array}$ \\
\hline & & $\begin{array}{l}\text { Expertise do Conselho de Ad- } \\
\text { ministração (ExpCA) }\end{array}$ & $\begin{array}{l}\text { Número de membros com graduação } \\
\text { ou pós-graduação em Ciências Con- } \\
\text { tábeis, Administração ou Economia. }\end{array}$ & $\begin{array}{l}\text { Formulário de re- } \\
\text { ferência - } \\
\text { item } 12.6 / 8 \\
\end{array}$ \\
\hline & $\begin{array}{l}\text { Qualidade da In- } \\
\text { formação Contá- } \\
\text { bil }\end{array}$ & $\begin{array}{l}\text { Qualidade da Informação } \\
\text { Contábil (QuIC) }\end{array}$ & $\begin{array}{l}\text { Resíduos da regressão (accruals dis- } \\
\text { cricionários) do modelo de gerencia- } \\
\text { mento de resultados de Kang e Siva- } \\
\text { ramakrishnan (1995). }\end{array}$ & Economática ${ }^{\circledR}$ \\
\hline
\end{tabular}

Fonte: Elaborado pelos autores.

Apresentado o constructo das variáveis que compõem o estudo, conforme Quadro 1, tem-se então, na sequência, o modelo de equação de regressão linear múltipla para testar os pressupostos de análise relacionados as hipóteses de pesquisa elaboradas no referencial teórico.

$$
\begin{gathered}
\text { MTB }=\beta_{0}+\beta_{1} \text { RepAud }+\beta_{2} \text { ComAud }+\beta_{3} H A u d+\beta_{4} H N A u d+\beta_{5} \text { TamCA }+\beta_{6} \text { GenCA }+\beta_{7} \text { DualD } \\
+\beta_{8} \text { ReunCA }+\beta_{9} \operatorname{Exp} C A+\beta_{10} Q u I C+\varepsilon
\end{gathered}
$$

Equação (2)

O procedimento de tabulação das variáveis foi executado por meio de planilha eletrônica. Posteriormente, para análise dos acrruals, fez-se uso da técnica estatística de regressão linear múltipla com uso do software estatístico SPSS 21®, extraindo os resíduos por caso, conforme modelo KS de Kang e Sivaramakrishnan (1995). Por fim, para verificar se existe influência das variáveis independentes de estrutura de auditoria, conselho de administração e qualidade da informação contábil no desempenho de mercado medido pelo indicador Market to Book Value, novamente fez-se uso da técnica estatística de regressão linear múltipla. Cabe destacar que, para o modelo final, foram observados os pressupostos da técnica estatística aplicada, a saber: normalidade, multicolinearidade, autocorrelação dos resíduos, heterogeneidade e omissão de variáveis relevantes. 


\section{RESULTADOS}

Previamente a análise de regressão, que indicará se há influência das variáveis independentes com a variável dependente do estudo, apresenta-se na Tabela 1 a estatística descritiva destas a fim de conhecimento da amostra. Cabe destacar que, três delas, sendo reputação da empresa de auditoria, comitê de auditoria e dualidade do diretor são dummies, determinando que estas são do tipo categóricas e, assim, serão apresentadas em termos percentuais. As demais variáveis são medidas através de índices (numéricas), conforme apresentado nos procedimentos metodológicos.

Tabela 1 - Estatística Descritiva das Variáveis Numéricas e Categóricas

\begin{tabular}{|c|c|c|c|}
\hline Variáveis Numéricas & \multicolumn{2}{|c|}{ Média } & Desvio-Padrão \\
\hline Market to Book Value & \multicolumn{2}{|c|}{2,0302} & 2,5130 \\
\hline Honorários de Auditoria & \multicolumn{2}{|c|}{0,0289} & 0,0289 \\
\hline Honorários de Não Auditoria & \multicolumn{2}{|c|}{0,0046} & 0,0147 \\
\hline Tamanho do Conselho de Administração & \multicolumn{2}{|c|}{0,8310} & 0,1759 \\
\hline Gênero do Conselho de Administração & \multicolumn{2}{|c|}{0,0743} & 0,1450 \\
\hline Reuniões do Conselho de Administração & \multicolumn{2}{|c|}{3,1407} & 2,0105 \\
\hline Expertise do Conselho de Administração & \multicolumn{2}{|c|}{1,6085} & 1,1339 \\
\hline Qualidade da Informação Contábil & \multicolumn{2}{|c|}{0,0535} & 0,0576 \\
\hline Variáveis Categóricas & Sim & Não & Total \\
\hline Reputação da Empresa de Auditoria & $80,74 \%$ & $19,26 \%$ & $100,00 \%$ \\
\hline Comitê de Auditoria & $22,47 \%$ & $77,53 \%$ & $100,00 \%$ \\
\hline Dualidade do Diretor & $24,44 \%$ & $75,56 \%$ & $100,00 \%$ \\
\hline
\end{tabular}

Fonte: Dados da pesquisa.

Mediante Tabela 1, infere-se que a variável dependente Market to Book Value apresenta média de 2,0302, o que pode ser considerado positivo, pois, em geral, o valor de mercado das empresas da amostra é superior ao dobro de seu valor contábil. Quanto as variáveis independentes numéricas, destaque para os honorários de auditoria pagos aos auditores independentes, onde, verifica-se que, em média, as empresas despendem anualmente o equivalente a 0,0289\% do valor contábil de seu ativo com estes serviços.

Além disso, em lógica similar, tem-se que com honorários de não auditoria, que representam principalmente os serviços de consultoria prestados por auditores independentes a seus clientes, as empresas despendem a proporção de $0,0046 \%$ de seu ativo total contábil no ano. Percentualmente, estes gastos podem ser considerados baixos quando comparados com os dispêndios em honorários de auditoria propriamente ditos e, sobretudo, em relação ao ativo total das organizações, mas em valores absolutos, os mesmos podem alcançar números expressivos.

Por fim, acerca da estatística descritiva das variáveis categóricas, destaque para a reputação da empresa de auditoria, apontando esta que a maioria (80,74\%) das organizações são auditadas por empresas com elevada reputação (no caso, big four). Visto isto, ademais, a fim de se atingir ao objetivo de pesquisa proposto, que perpassa por verificar a influência da estrutura de auditoria, conselho de administração e qualidade da informação contábil no índice Market to Book Value de empresas brasileiras listadas na B3, fez-se uso da regressão linear múltipla, sendo os resultados apresentados na Tabela 2 para posterior análise. 
Tabela 2 - Resultados da Regressão Múltipla

\begin{tabular}{|c|c|c|c|c|}
\hline \multirow{2}{*}{ Variáveis } & \multicolumn{3}{|c|}{ Coeficientes } & \multirow{2}{*}{$\begin{array}{c}\text { Estat. Colinearidade } \\
\text { VIF }\end{array}$} \\
\hline & Coef. & $t$ test & Sig. & \\
\hline RepAud & 0,997 & 4,11 & $0,000^{*}$ & 1,12 \\
\hline ComAud & $-0,531$ & $-2,16$ & $0,032^{* *}$ & 1,11 \\
\hline HAud & $-5,610$ & $-1,54$ & 0,124 & 1,21 \\
\hline HNAud & 31,525 & 2,70 & $0,007^{*}$ & 1,07 \\
\hline TamCA & 0,212 & 0,25 & 0,800 & 1,48 \\
\hline GenCA & 1,153 & 1,38 & 0,169 & 1,03 \\
\hline DualD & $-0,384$ & $-1,78$ & $0,075^{* * *}$ & 1,02 \\
\hline ReunCA & 0,048 & 0,36 & 0,718 & 1,10 \\
\hline ExpCA & $-0,023$ & $-0,43$ & 0,666 & 1,37 \\
\hline QuIC & 7,292 & 1,88 & $0,060^{* * *}$ & 1,04 \\
\hline (Constante) & 0,802 & 1,07 & 0,285 & - \\
\hline $\mathrm{R}^{2}$ & \multicolumn{4}{|c|}{0,1009} \\
\hline Significância Modelo & \multicolumn{4}{|c|}{0,0000} \\
\hline Jarque-Bera & \multicolumn{4}{|c|}{0,0000} \\
\hline Durbin-Watson & \multicolumn{4}{|c|}{1,8782} \\
\hline White Test & \multicolumn{4}{|c|}{0,0000} \\
\hline Reset de Ramsey & \multicolumn{4}{|c|}{0,0969} \\
\hline $\mathrm{N}^{\circ}$ Observações & \multicolumn{4}{|c|}{405} \\
\hline
\end{tabular}

Legenda: RepAud - Reputação da Auditoria Independente; ComAud - Presença do Comitê de Auditoria; HAud - Honorários Pagos de Auditoria; HNAud - Honorários Pagos de Não Auditoria; TamCA - Tamanho do Conselho de Administração; GenCA - Gênero do Conselho de Administração; DualD - Dualidade do Diretor; ReunCA - Reuniões do Conselho de Administração; ExpCA - Expertise do Conselho de Administração; QuIC - Qualidade da Informação Contábil.

Significância das Variáveis: *Sig. ao nível de 1\%; **Sig. ao nível de 5\%; *** Sig. ao nível de $10 \%$.

Fonte: Dados da pesquisa.

Conforme Tabela 2, é possível observar, inicialmente, que o modelo é significativo ao nível de $5 \%$, o que permite inferências. É imprescindível destacar que este grau de confiabilidade também foi adotado para os pressupostos do modelo. Neste sentido, o teste de Jarque-Bera aponta que não há normalidade nos resíduos. Todavia, devido ao número de observações expressivas, estes foram relaxados conforme teorema central do limite. Quanto a multicolinearidade, o VIF (Variance Inflation Factor) máximo foi de 1,48, inferior ao nível de 5 aceito pela literatura, não havendo, portanto, problemas deste tipo.

Ademais, o teste de Durbin-Watson apresentou resultado de 1,8782, estando entre o aceitável, que varia de 1 a 3 (e próximo ao ideal que é 2), apontando que o modelo não contém problemas de autocorrelação. Ademais, o White Test sugere que o modelo apresenta problemas de heterocedasticidade, todavia, estes foram corrigidos com a aplicação da regressão linear múltipla robusta, que conforme Fávero, Belfiore, Silva e Chan (2009) insere a matriz White ajustando os erros padrão em função da heterocedasticidade do modelo (Fávero et al., 2009). Por fim, o Reset de Ramsey aponta que não há problemas de omissão de variáveis relevantes no modelo, ou seja, este está bem especificado (quanto suas variáveis explicativas). Pela acuidade e singularidade deste último, destaca-se, conforme Gujarati (2006) de forma teórica e Costa e Costa (2018) em estudo empírico, que valores superiores a 0,05 significam a ausência do problema a 5\% de significância.

Em face aos resultados, tem-se, num primeiro momento, que três das quatro variáveis vinculadas a estrutura de auditoria são significativas, seja ao nível de $1 \%$ ou $5 \%$. Mais especificamente, a reputação da empresa de auditoria independente está positivamente relacionada ao Market to Book Value. Assim, organizações auditadas por firmas big four de auditoria possuem maior desempenho de mercado, corroborando com o entendimento teórico de Lameira (2007) e Collin et al. (2013), que destacam que as maiores auditorias possuem subsídios para prestar um serviço de maior qualidade 
e, consequentemente, seus clientes tem seu desempenho maximizado. Além disso, este achado corrobora com o resultado empírico de Al Farooque et al. (2007) em estudo com empresas de Blangladesh. Deste modo, se aceita a hipótese 1 do estudo de que a reputação da empresa de auditoria independente influência positivamente no desempenho de mercado das empresas brasileiras em estudo.

Quanto à presença de comitê de auditoria nas organizações, percebe-se que a mesma está negativamente relacionada com o indicador Market to Book Value, o que conota que na amostra em estudo, empresas que possuem comitê de auditoria apresentam desempenho de mercado inferior. Tal relação encontrada diverge do esperado, pois conforme Christensen et al. (2010) e Azim (2012), o comitê de auditoria é um mecanismo com potencial para diminuir problemas, como nos relatórios financeiros das empresas e proporciona transparência, o que deveria contribuir positivamente para o desempenho das organizações (Politelo, 2013). Desta forma, a hipótese 2 é rejeitada, pois a relação entre a presença do comitê de auditoria e o índice Market to Book Value encontrada é inversa dos pressupostos identificados na literatura, o que conota, por consequência, que o mercado pode identificar o comitê de auditoria apenas como algo burocrático, não compreendendo seu real papel, que deve ser disseminado pelas organizações para melhor compreensão das partes interessadas e, assim, maximizando sua utilidade.

Na sequência, têm-se a variável honorários de auditoria que se apresenta negativamente relacionada ao Market to Book Value. Este fato leva ao entendimento que empresas com maior desempenho de mercado despendem numerário inferior com auditoria na proporção de seu ativo total comparativamente a aquelas em dificuldades. Este achado corroboraria com os estudos de Haat $e t$ al. (2008) em empresas da Malásia e Moutinho et al.(2012) para corporações dos Estados Unidos, que também encontraram relação inversa entre honorários de auditoria e desempenho, todavia, para o cenário brasileiro pesquisado, apesar de negativo, o coeficiente não é significativo, o que não permite inferências e, consequentemente, rejeita-se a hipótese 3a.

A última variável vinculada à estrutura de auditoria proposta está pautada nos honorários pagos pelas organizações aos auditores independentes referentes a serviços de não auditoria, compreendendo majoritariamente por consultoria prestada aos clientes. Assim, nota-se pela regressão que quanto maior o gasto das organizações com serviços de consultoria prestados por seus auditores, maior é o desempenho de mercado da organização. De fato, conforme Kubr (1986), serviços de consultoria apresentam potencial para a implantação de medidas adequadas à realidade das organizações e, assim, proporcionam desempenho maior as mesmas. Em suma, considerado tais serviços de forma isolada, estes têm contribuído positivamente para o desempenho das corporações. Assim, se aceita a hipótese $3 \mathrm{~b}$ de que há relação, no caso positiva, entre honorários de não auditoria e o índice Market to Book Value.

Procurou-se, na sequência, testar à relação de cinco variáveis vinculadas ao conselho de administração com o índice Market to Book Value das organizações brasileiras. Todavia, destas, apenas a dualidade do diretor apresentou poder explicativo, ao nível de $10 \%$ de significância, a fim de explicar o Market to Book Value das empresas brasileiras. Mais especificamente, a variável dualidade do diretor influencia negativamente o índice Market to Book Value. Assim, infere-se que quando o CEO também exerce a função de presidente do conselho de administração, as empresas tem seu desempenho de mercado mitigado. Esta relação converge com o estudo de Ehikioya (2009), que encontrou relação negativa entre a dualidade de função do CEO e o desempenho de organizações nigerianas. Com base no achado, o autor (2009) enfatiza que para a obtenção de desempenho superior, existe a necessidade de separar a posição do CEO e do presidente, garantindo inclusive a independência do conselho. Destaca-se, assim, que a hipótese 6 é aceita, visto que os resultados encontrados confirmam relação negativa entre a dualidade do diretor e o desempenho de mercado nas empresas brasileiras estudadas. 
Ademais, evidenciou-se que o tamanho do conselho de administração, o gênero dos membros que compõem tal conselho, a frequência de reuniões existente e a expertise do conselho de administração não possuem relação significante com a variável dependente Market to Book Value. Tal fato conota que não é possível fazer inferenciais sobre tais variáveis no que tange a amostra de estudo. Logo, destaca-se que as hipóteses de pesquisa 4, 5, 7 e 8 são rejeitadas devido à falta de significância estatística entre tais variáveis independentes e a variável dependente Marlet to Book Value. Cabe destacar que, conforme Valenti, Luce e Mayfield (2011), a falta de significância pode ocorrer devido às medidas das variáveis, que diante da complexidade do fenômeno observado, podem não levar em conta todos os aspectos necessários ou, ainda, que realmente não há relação entre tais variáveis nas empresas brasileiras.

Por fim, quanto à qualidade da informação contábil, nota-se que esta é significativa ao nível de $10 \%$, com coeficiente positivo, determinando que quanto maior o grau de gerenciamento de resultados, o desempenho de mercado das organizações também é maximizado. Desta forma, por haver influência da qualidade da informação contábil com o índice Market to Book Value, a hipótese 10 de pesquisa é aceita. Cabe destacar que, os accruals determinados pelo modelo de gerenciamento de resultados KS estão em valores absolutos, visto que o objetivo é verificar a qualidade da informação contábil e, assim, o fato das organizações gerenciarem seus resultados de forma negativa ou positiva não possui funcionalidade, conforme entendimento extraído de Krishnan (2003).

Por fim, destaca-se a relevância deste resultado, pois aponta que empresas com melhor qualidade na informação contábil possuem menor desempenho no que tange ao índice Market to Book Value. Neste ínterim, Watts e Zimmerman (1981) alertam ao aspecto de que é improvável pressupor que auditores são impecavelmente independentes de seus clientes e que uma das formas mais comuns de abalar a independência do auditor deriva-se da perspectiva deste de auferir futuras rendas da relação com seu cliente. Logo, uma possível explicação para a relação evidenciada entre a qualidade da informação contábil e o índice Market to Book Value seria de que auditores são mais flexíveis com clientes que possuem alto desempenho, permitindo maior nível de gerenciamentos de resultados de acordo com as necessidades dos mesmos por visualizarem maiores benefícios futuros na prestação de serviços a estes e, assim, tendem aceitar imposições de seus clientes para não correrem o risco de serem trocados por firma de auditoria independente concorrente.

\section{CONCLUSÃO}

Este estudo teve por objetivo verificar a influência da estrutura de auditoria, conselho de administração e qualidade da informação contábil no índice Market to Book Value de empresas brasileiras listadas na B3. Os resultados apontam que as três dimensões analisadas influenciam no desempenho de mercado das organizações brasileiras, corroborando com as perspectivas teóricas e empíricas de diversos autores.

De forma específica, quanto a estrutura de auditoria, verificou-se que a reputação da empresa de auditoria independente (big four vs não big four) e os honorários pagos aos auditores independentes referente aos serviços de não auditoria influenciam positivamente o desempenho de mercado das organizações, enquanto a presença de comitê de auditoria nas organizações tem relação negativa com o índice Market to Book Value. A interpretação destes resultados por parte de gestores é essencial, visto que sua correta utilização no âmbito organizacional possui capacidade de maximizar o valor das organizações, foco primordial no mundo empresarial.

Para as variáveis do conselho de administração testadas, constatou-se que apenas uma delas possui influência com o desempenho de mercado das organizações, sendo a dualidade do diretor e de forma negativa, convergente com a literatura. Porém, para a amostra em estudo, tamanho do conselho de administração, gênero do conselho de administração, número de reuniões do conselho 
de administração e expertise do conselho de administração não apresentaram significância, sugerindo não influenciar o índice Market to Book Value no Brasil. Logo, destaque é dado para a observância do CEO da organização não ser também o presidente do conselho de administração, pois o mercado entende tal prática de forma negativa e penaliza o valor da empresa diante disto.

Por último, ainda tem-se que a qualidade da informação contábil, medida pelos accruals discricionários (em termos absolutos) derivados do modelo de gerenciamento de resultados KS de Kang e Sivaramakrishnan (1995) possuem relação positiva com o índice Market to Book Value, ou seja, empresas que gerenciam mais resultados possuem maior desempenho de mercado. Isto pode ocorrer devido a clientes com maior poder econômico-financeiro terem representatividade superior na carteira de clientes das firmas de auditoria independente e, principalmente, demonstrarem ser bons parceiros para os auditores auferir futuras rendas, mitigando a independência destes que, por consequência, aceitam imposições de seus clientes que acarretam na diminuição da qualidade da informação contábil. Desta forma, sugere-se ao mercado como um todo precisa ficar mais atento à qualidade da informação contábil das organizações.

No geral, os resultados sugerem que a estrutura de auditoria, conselho de administração e qualidade da informação contábil devem ser aspectos considerados pelos diversos stakeholders que se ligam as organizações para uma tomada de decisão coerente. Todavia, apesar de importantes, estes achados não podem ser generalizados como realidade de todas as empresas brasileiras e, assim, devem ser interpretados com cautela. Tem-se ainda que, observações cobrindo período de três anos e no contexto de um único país não podem fornecer conclusões finais para o comportamento das variáveis, necessitando estudos futuros abrangendo períodos longitudinais de tempo e também que se estendam a outros contextos. Além disso, o poder explicativo do modelo operacionalizado neste estudo (apesar de corretamente especificado, conforme teste de Reset de Ramsey) aponta que outros fatores devem influenciar o valor de mercado das empresas como, por exemplo, de cunho comportamental - excesso de confiança de investidores no mercado de capitais, entre outros - e, assim, a exploração destes em pesquisas posteriores é primordial.

Desta forma, para estudos futuros, recomenda-se o uso de outros indicadores que medem o desempenho de mercado das organizações, como o $Q$ de Tobin e o retorno das ações, muito usual em pesquisas na área de finanças corporativas e, ainda, pouco difundido em estudos de auditoria no âmbito nacional. Por fim, pode-se ainda considerar outras variáveis de estrutura de governança corporativa como, por exemplo, relatório financeiro divulgado em tempo hábil, tamanho do comitê de auditoria, comitê de remuneração, comitê de nomeação, etc., todas estas existentes na literatura da área.

\section{REFERÊNCIAS}

Al Farooque, O., Van Zijl, T., Dunstan, K., \& Karim, AKM W. (2007). Corporate governance in Bangladesh: link between ownership and financial performance. Journal Compilation, 15(6), 1453-1468.

Almeida, A. M., Santos, J. F., Ferreira, L. F. V. M., \& Torres, F. J. V. (2010). Evolução da qualidade das práticas de governança corporativa: um estudo das empresas brasileiras de capital aberto não listadas em bolsa. Revista de Administração Contemporânea, 14(5), 907-924.

Almeida, J. E. F., \& Almeida, J. C. G. de. (2009). Auditoria e Earnings Management: estudo empírico nas empresas abertas auditadas pelas big four e demais firmas de auditoria. Revista Contabilidade e Finanças, 20(50), 62-74. 
Almeida, J. E. F., Lopes, A. B., \& Corrar, L. J. (2011). Gerenciamento de resultados para sustentar a expectativa do mercado de capitais: impactos no índice market-to-book. Advances in Scientific and Applied Accounting, 4(1), 44-62.

Azim, M. I. (2012). Corporate governance mechanisms and their impact on company performance: a structural equation model analysis. Australian Journal of Management, 37(3), 481-505.

Campbell, K., \& Minguez-Vera, A. (2008). Gender diversity in the boardroom and firm financial performance. Journal of Business Ethics, 83(1), 435-451.

Chen, L., \& Zhao, X. (2006). On the Relation between the Market-to-Book Ratio, Growth Opportunity, and Leverage Ratio. Finance Research Letters, 3(4), 253-266.

Christensen, J., Kent, P., \& Stewart, J. (2010). Corporate governance and company performance in Australia. Australian Accounting Review, 20(4), 372-386.

Collin, S-O. Y., Smith, E., Umans, T., Broberg, P., \& Tagesson, T. (2013). Mechanisms of corporate governance going international: Testing its performance effects in the Swedish economy, 2004. Baltic Journal of Management, 8(1), 79-101.

Correia, L. F., Amaral, H. F., \& Louvet, P. (2011). Um índice de avaliação da qualidade da governança corporativa no Brasil. Revista Contabilidade E Finanças, 22(55), 45-63.

Costa, L. F. C. da, \& Costa, M. G. da. (2018). Os Impactos de Fatores Macroeconômicos sobre Índices de Ações Setoriais: uma Análise Através do Algoritmo de Seleção de Modelos Autometrics. Revista Evidenciação Contábil \& Finanças, 6(3), 96-109.

Davidson, R., Stewart, J. G., \& Kent, P. (2005). Internal governance structure and earnings management. Accounting and Finance, 45(2), 241-267.

DeAngelo, L. E. (1981). Auditor Independence, 'Low Balling', and Disclosure Regulation. Journal of Accounting and Economics, 3(2), 113-127.

Dickins, D. E., Higgs, J. L., \& Skantz, T. R. (2008). Estimating Audit Fees Post-SOX. Current Issues in Auditing, 2(1), 9-18.

Ehikioya, B. I. (2009). Corporate governance structure and firm performance in developing economies: evidence from Nigeria. Corporate Governance: The International Journal of Business in Society, 9(3), 231-243.

Fávero, L. P., Belfiore, P., Silva, F. L. da, \& Chan, B. L. (2009). Análise de dados-Modelagem Multivariada para Tomada de Decisões. (2. ed.). Rio de Janeiro: Elsevier.

Firth, M. (1997). An analysis of the stock market performance of new issues in New Zealand. PacificBasin Finance Journal, 5(1), 63-85.

Francis, J. (1984). The effect of audit firm size on audit prices. Journal of Accounting and Economics, 6(2), 133-151.

Gaur, S. S., Bathula, H., \& Singh, D. (2015). Ownership concentration, board characteristics and firm performance: a contingency framework. Management Decision, 53(5), 911-931.

Gilio, L.(2010). Análise da capacidade explicativa de informações contábeis para o índice market-to-book de empresas listadas no IBOVESPA. Congresso USP de controladoria e Contabilidade, São Paulo/SP.

Gujarati, D. N. (2006). Econometria Básica. (4. ed.). Rio de Janeiro: Elsevier. 
Haat, M. H. C., Rahman, R. A., \& Mahenthiran, S. (2008). Corporate governance, transparency and performance of Malaysian companies. Managerial Auditing Journal, 23(8), 744-778.

Hallak, R. T. P., \& Silva, A. L. C. da. (2012). Determinantes das Despesas com Serviços de Auditoria e Consultoria Prestados pelo Auditor Independente no Brasil. Revista Contabilidade e Finanças, 23(60), 223-231.

Healy, P. M., \& Wahlen, J. M. (1999). A review of the earnings management literature and its implications for standard setting. Accounting Horizons, 13(4), 365-383.

Johl, S. K., Kaur, S., \& Cooper, B. J. (2015). Board Characteristics and Firm Performance: Evidence from Malaysian Public Listed Firms. Journal of Economics, Business and Management, 3(2), 239-243.

Kang, S. H., \& Sivaramakrishnan, K. (1995). Issues in testing earnings management: an instrumental variable approach. Journal of Accounting Research, 33(2), 353-367.

Krishnan, G. V. (2003). Does big 6 auditor industry expertise constrain earnings management? Accounting Horizons, 17(1), 1-16.

Kubr, M. (1986). Consultoria um guia para a profissão. (1. ed.). Rio de Janeiro: Editora Guanabara Koogan S.A.

Lameira, V. de J. (2007). Governança corporativa, risco e desempenho das companhias abertas brasileiras uma análise do relacionamento entre as práticas de governança corporativa, o risco e o desempenho das companhias abertas brasileiras. Tese (Doutorado em Administração). Pontifícia Universidade Católica do Rio de Janeiro, Rio de Janeiro/RJ.

Lopes, A. B \& Iudícibus, S. de. (2012). Teoria avançada da contabilidade. (2. ed.). São Paulo/SP: Editora Atlas.

Martinez, A. L. (2001). 'Gerenciamento' de resultados contábeis: estudo empírico das companhias abertas brasileiras. Tese (Doutorado em Ciências Contábeis). Departamento de Contabilidade e Atuária, Universidade de São Paulo: São Paulo/SP.

Martinez, A. L. (2008). Detectando Earnings Management no Brasil: estimando os Accruals Discricionários. Revista Contabilidade E Finanças, 19(46), 7-17.

Martinez, A. L. (2010). Quando o Conselho de Administração e a Auditoria evitam o Gerenciamento de Resultados? Evidências Empíricas para Empresas Brasileiras. Revista de Informação Contábil, $4(1), 76-93$.

Martinez, A. L., \& Moraes, A. de J. (2014). Association Between Independent Auditor Fees and Firm Value: A Study of Brazilian Public Companies. Journal of Modern Accounting and Auditing, 10(4), $442-450$.

Moutinho, V., Cerqueira, A., \& Brandão, E. (2012). Audit fees and firm performance. Working Paper Series.

Nascimento, A. M., \& Reginato, L. (2008). Divulgação da informação contábil, governança corporativa e controle organizacional: uma relação necessária. Revista Universo Contábil, 4(3), 25-47.

Ntim, C. G., \& Osei, K. A. (2011). The Impact of Corporate Board Meetings on Corporate Performance in South Africa. African Review of Economics and Finance, 2(2), 83-103.

Oliveira, K. P. S. de, Paulo, E. \& Martins, O. S. (2013). As relações entre a assimetria de informação e a governança corporativa no mercado de capitais brasileiro. Registro Contábil, 4(3), 56-70. 
Paulo, E., \& Leme, J. R. (2009). Gerenciamento de resultados contábeis e o anúncio dos resultados contábeis pelas companhias abertas brasileiras. Revista Universo Contábil, 5(4), 27-43.

Politelo, L. (2013).Mecanismos de Governança Corporativa e Desempenho de Empresas Familiares listadas na BMEFBovespa. Dissertação (Mestrado em Ciências Contábeis). Universidade Regional de Blumenau, Blumenau/SC.

Ramos, G. M., \& Martinez, A. L. (2006). Governança Corporativa e Gerenciamento de Resultados Contábeis. XXIX Encontro da Associação Nacional dos Programas de Pós-Graduação em Administração, Salvador/BA.

Silveira, A. D. M. A., \& Barros, L. A. B. de C. (2008). Determinantes da qualidade da governança corporativa das companhias abertas brasileiras. Revista Eletrônica de Administração, 14(3), 1-29.

Silveira, A. D. M. da, Barros, L. A. B. de C., \& Famá, R. (2006). Atributos Corporativos, Qualidade da Governança Corporativa e Valor das Companhias Abertas no Brasil. Revista Brasileira de Finanças, 4(1), 1-30.

Silveira, A.D. M. da, Barros, L. A. B. de C., \& Famá, R. (2003). Estrutura de governança e desempenho financeiro nas companhias abertas brasileiras: um estudo empírico. Caderno de Pesquisas em Administração, 10(1), 57-71.

Sousa. E. (2014). Market-to-Book: uma Avaliação das Companhias Listadas nos Segmentos Diferenciados da BM\&FBOVESPA. Brazilian Journal of Quantitative Methods Applied to Accounting - Contabilometria, 1(1), 56-67.

Ujunwa, A. (2012). Board characteristics and the financial performance of Nigerian quoted firms. Corporate Governance: The international journal of business in society, 12(5), 656-674.

Valenti, M. A., Luce, R., \& Mayfield, C. (2011). The Effects of Firm Performance on Corporate Governance. Management Research Review, 34(3), 266-283.

Wahba, H. (2015). The joint effect of board characteristics on financial performance: Empirical evidence from Egypt. Review of Accounting and Finance, 14(1), 20-40.

Watts, R. L., \& Zimmerman, J. L. (1981). The Markets for Independence and Independent Auditors. Working Paper Series, University of Rochester.

Whisenant, S., Sankaraguruswamy, S., \& Raghunandan, K. (2003). Evidence on the Joint Determination of Audit and Non-Audit Fees. Journal of Accounting Research, 41(4), 721-744. 